\title{
Use of Pre-Infusion Serum Uric Acid Levels as a Biomarker for Infusion Reaction Risk in Patients on Pegloticase
}

\author{
Robert T. Keenan · Herbert S. B. Baraf · Brian LaMoreaux (ID
}

Received: February 11, 2019 / Published online: March 14, 2019

(C) The Author(s) 2019

\begin{abstract}
Introduction: The aim of this work is to evaluate the impact of the timing of pre-infusion serum uric acid (sUA) test results for use in applying stopping rules for pegloticase to identify patients at risk for infusion reactions (IRs) while on therapy.

Methods: Data from the phase 3 clinical trials of pegloticase were reviewed and individual uric acid levels of the 85 patients who received the approved regimen of biweekly infusions were examined in relation to the occurrence of IRs.

Results: Of the 22 patients (26\%) who experienced an IR on pegloticase therapy without uric acid stopping rules, only seven (8\%) would have had IRs if pegloticase therapy had been discontinued after two consecutive pre-infusion sUA levels above $6 \mathrm{mg} / \mathrm{dl}$. If pegloticase therapy was
\end{abstract}

Enhanced Digital Features To view enhanced digital features for this article go to: https://doi.org/10.6084/ m9.figshare.7775984.

\section{R. T. Keenan}

Division of Rheumatology and Immunology,

Department of Medicine, Duke University School of

Medicine, Durham, NC, USA

H. S. B. Baraf

Arthritis and Rheumatism Associates, George

Washington University, Wheaton, MD, USA

B. LaMoreaux $(\bowtie)$

Horizon Pharma, Lake Forest, IL, USA

e-mail: blamoreaux@horizonpharma.com stopped after a single pre-infusion sUA above $6 \mathrm{mg} / \mathrm{dl}$, only two patients (2\%) would have experienced IRs during the clinical studies.

Conclusions: A pre-infusion sUA level functions as a highly accurate biomarker for identification of patients who are at risk of IRs while on pegloticase therapy. Stopping pegloticase in patients who have a rise in pre-infusion uric acid levels to above $6 \mathrm{mg} / \mathrm{dl}$ while on therapy would result in most IRs being avoided.

Funding: Horizon Pharma.

Keywords: Infusion reactions; Pegloticase; Refractory gout; Stopping rules; Uncontrolled gout; Uric acid

\section{INTRODUCTION}

Pegloticase is currently the only therapy approved in the United States for gout refractory to conventional therapy or uncontrolled gout. As a biologic medication, pegloticase is administered intravenously every 2 weeks in contrast to oral urate lowering therapies (ULT). The optimal duration of pegloticase treatment has not been established.

The approval of pegloticase in 2011 was based on two replicate blinded randomized phase 3 trials (C0405 and C0406) enrolling 212 patients in three cohorts. Eighty-five patients were treated with pegloticase infusions every 2 weeks, 84 patients with pegloticase alternating 
with placebo (sterile phosphate buffered saline) every 2 weeks, and 43 patients with placebo every 2 weeks. Patients in all three cohorts received the same pre-infusion medications. The primary study outcome was the proportion of patients in each treatment group who had uric acid levels of $<6 \mathrm{mg} / \mathrm{dl}$ at $80 \%$ or more of the 16 separate uric acid measurements during months 3 and 6 of the trial. Patients meeting this criterion were designated as uric acid responders. Those patients who did not meet this endpoint were designated as non-responders. Forty-two percent of patients in the pegloticase every 2 week cohort met this strict primary endpoint (uric acid responders). There were no responders among patients in the placebo cohort. Although plasma uric acid levels were used as the primary determinate in the phase 3 studies, concurrent serum uric acid (sUA) levels were drawn and the two methods had high agreement [1]. Based on these results, pegloticase was approved for use given as infusions every 2 weeks. Importantly, but not included as part of the primary endpoint, uric acid levels were checked with every pegloticase infusion throughout the entire 6-month trial and were blinded to the investigators until the trials concluded.

When the phase 3 clinical trials of pegloticase were being conducted, it was not appreciated that uric acid levels while on therapy could reflect the development of anti-drug antibodies to pegloticase. These anti-pegloticase antibodies can cause accelerated clearance of pegloticase leading to a reduced systemic pegloticase level, loss of uric acid-lowering effect, and a higher risk of infusion reactions (IRs) [2]. As these effects were unanticipated at the time of the pivotal trials, guidelines for discontinuing pegloticase based on sUA levels while on therapy were not established. Only after a post hoc analysis of the phase 3 data did the need for such guidelines become apparent. Thus, the non-responder group of patients in the trialsthose whose uric acid levels increased to above $6 \mathrm{mg} / \mathrm{dl}$ while on treatment-continued to be treated throughout the duration of the trial with pegloticase.

The most common adverse events in the phase 3 pegloticase trials were gout flares and
IRs. Gout flares occurred in $77 \%$ of patients treated with pegloticase every 2 weeks and 81\% of patients receiving placebo infusions. IRs occurred in 22 patients or $26 \%$ of subjects receiving pegloticase every 2 weeks compared to two patients or $5 \%$ of subjects receiving placebo infusions.

After the trials were completed, Baraf et al. published a post hoc analysis examining the uric acid levels of patients while on pegloticase and their relationship to the occurrence of IRs. In this analysis, 20 of the 22 patients who experienced IRs (91\%) had an sUA level greater than $6 \mathrm{mg} / \mathrm{dl}$ at the time of the infusion that resulted in an IR. In contrast, among those 22 patients experiencing IRs, just one had a level of less than $6 \mathrm{mg} / \mathrm{dl}$ at the time of the infusion (5\%). Additionally, one patient had a first-dose IR (5\%) [3]. When viewed in terms of all treated patients in the pivotal trials dosed with pegloticase every 2 weeks, $1 / 85$ or $1 \%$ of patients had a first-dose IR.

The uric acid levels used by Baraf et al. in their retrospective analysis were drawn at the time of each pegloticase infusion, and then the information obtained from those determinations while on therapy was applied to the subsequent infusion 2 weeks later to conclude if therapy should be discontinued. Obtaining uric acid levels at the time of pegloticase infusions remains logistically easier, but having a uric acid level obtained prior to administration of pegloticase provides current and immediately actionable information regarding patients' ongoing response to therapy. This publication provided insight into the importance of pretreatment sUA determinations in patients on pegloticase so as to identify patients at risk of IRs. This key modification in monitoring has become integral in developing stopping rules designed to improve patient safety with chronic pegloticase therapy.

The Baraf analysis provides the potential reduction of IRs by checking sUA levels at the time of each infusion and then using that level 14 days later to determine whether subsequent infusions are given. Checking sUA levels immediately or one to 2 days before each pegloticase infusion allows the decision to continue or stop therapy to happen just prior to 
each infusion. The purpose of this analysis was to quantify the reduction in IRs if pre-treatment sUA levels were checked 1-2 days before pegloticase infusions subsequent to the initial infusion.

\section{METHODS}

Data and information on IRs were obtained from the two replicate, 6-month randomized, double-blind, placebo-controlled phase 3 trials of pegloticase (labeled C0405 and C0406, identifier NCT00325195) as well as the open-label extension study (C0407; NCT01356498) that followed. The study design for these has been previously described, and the focus of this paper is on the experience of the patients dosed with the FDA-approved every 2-week administration of pegloticase $[1,4]$. This article is based on previously conducted studies and does not contain any studies with human participants performed by any of the authors.

In brief, patients with uncontrolled gout who were either intolerant of or refractory to oral uric acid-lowering therapy were randomized to receive pegloticase every 2 weeks, every 4 weeks, or placebo infusions. All patients received a pre-infusion regimen of oral fexofenadine and acetaminophen as well as intravenous hydrocortisone $200 \mathrm{mg}$ immediately before each pegloticase administration. Eightyfive patients were treated with pegloticase every 2 weeks and 43 patients received placebo infusions during the 6-month parallel trials. Prior to initiating pegloticase therapy, patients discontinued other urate-lowering therapies and were provided with either non-steroidal anti-inflammatory drugs (NSAIDs) or colchicine for gout flare prophylaxis throughout the trials. All study protocols had institutional review board (IRB) approval and patients provided informed consent before entrance into the studies.

The clinical trial primary endpoint for efficacy was the proportion of patients who had plasma uric acid (pUA) levels below $6 \mathrm{mg} / \mathrm{dl}$ for 13 or more of the 16 uric acid blood draws during months 3 and 6 . Any patients who withdrew from the trial for any reason or had fewer than $80 \%$ of their pUA levels below $6 \mathrm{mg}$ / $\mathrm{dl}$ were classified as non-responders for the primary efficacy endpoint. Secondary endpoints included tophus resolution, gout flares, swollen and tender joint counts, and other patient-reported quality-of-life outcomes.

IRs were defined in the study protocol as "any otherwise unexplained adverse event or cluster of temporally related events occurring during or within $2 \mathrm{~h}$ of infusion" [3]. Each IR triggered a standardized evaluation including serial vital signs, clinical history, focused clinical examination, 12-lead electrocardiogram, and specific laboratory tests.

Patients had uric acid levels checked with every infusion throughout the trials that were blinded to the investigator. Baraf et al. subsequently analyzed in a post hoc manner the effect of viewing the uric acid levels at the time of each infusion and stopping future infusions of pegloticase based on having a rising uric acid level. This was done to determine if stopping treatment prospectively would alter IR risk or subsequent responder status. As pUA and sUA levels had a high degree of correlation, both the Baraf et al. and the present study used sUA levels in the analyses. Again, this analysis assumed that a uric acid level would be drawn with each pegloticase infusion and that the result of that uric acid draw would not be available until after the corresponding infusion completed. Under this premise, any given uric acid level would not be viewable and actionable until the next pegloticase infusion 2 weeks later.

However, if patients receiving therapy with pegloticase could have a uric acid levels drawn 24-48 h before each pegloticase infusion (not including the first infusion), it would make the uric acid level result available to inform the impending dose (Fig. 1).

With the updated assumption that obtaining pre-infusion uric acid levels is possible while on pegloticase therapy, the uric acid data with each infusion from the 6-month clinical trials were re-analyzed in the patients dosed every 2 weeks. The effect of having actionable pre-infusion uric acid levels on occurrence of IRs and drug efficacy were computed and reported as if these results were available to the treating physician immediately prior to each infusion. These results were then compared to the results 
A Original Analysis

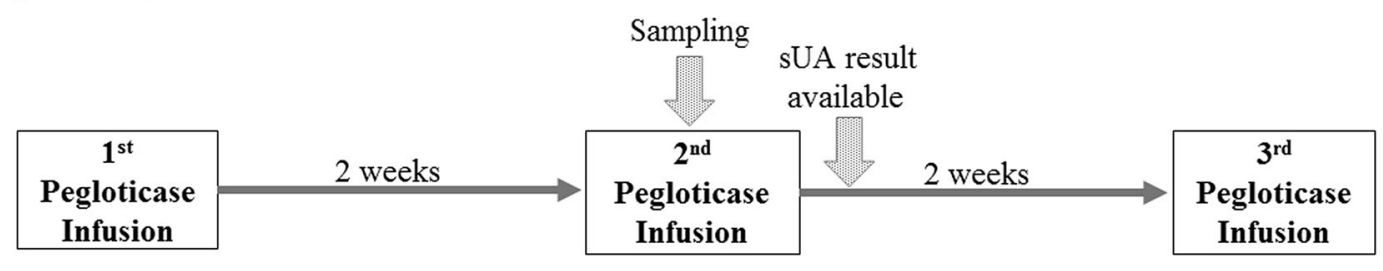

B Current Clinical Paradigm

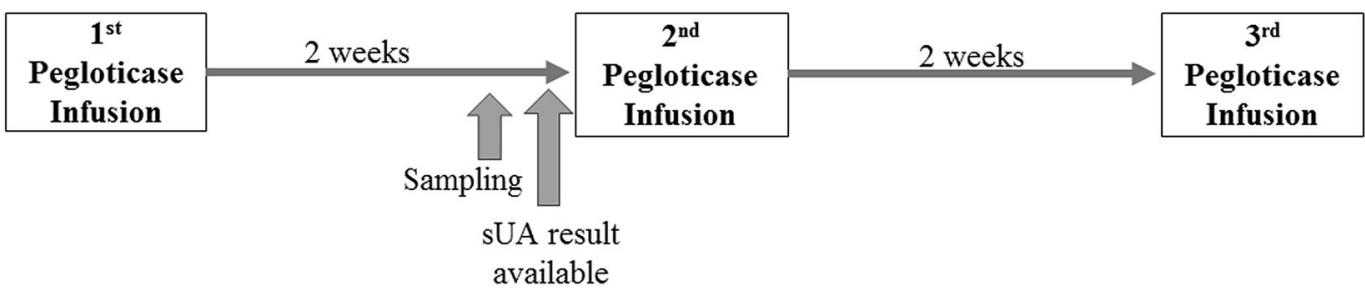

Fig. 1 Testing uric acid levels during infusions (checkered arrows) yields results available after infusions are complete, informing the infusion 2 weeks later. Testing uric acid

obtained by Baraf et al. with respect to safety (IRs) and efficacy (proportion of responders), where the decision to stop infusions after one or two consecutive sUAs above $6 \mathrm{mg} / \mathrm{dl}$ was made 2 weeks after sUA levels were drawn [3].

\section{RESULTS}

In the 6-month phase 3 RCTs, 85 patients were dosed with pegloticase every 2 weeks and 43 patients received placebo infusions. Without stopping rules in place regarding uric acid levels, 22 of the 85 patients dosed with pegloticase (26\% of patients) experienced a total of 43 IRs among 852 infusions (5\% of infusions).

Among patients experiencing IRs, 97\% occurred during pegloticase infusions and 3\% occurred during the 2 -h post-infusion observation period. The most common pegloticase-associated IR symptoms (occurring in $>5 \%$ of patients with IRs) included chest discomfort, flushing, dyspnea, back pain, hyperhidrosis, nausea, erythema, urticaria, chest pain, pruritus, rash, muscle spasms, and abdominal pain.

This analysis shifts the view of sUA levels from being drawn during each pegloticase infusion and not actionable until the infusion levels prior to infusions (gray-filled arrows) yields results available prior to infusions, informing the impending infusion

2 weeks later to being drawn and viewed 24-48 h prior to each infusion after the first, and actionable immediately before the next infusion now labeled "pre-infusion" sUA levels (see Fig. 1).

With this change, stopping after two consecutive pre-infusion sUA levels of above six would have resulted in the administration of only 620 of the 852 infusions given in the original trial. With these infusions avoided, seven patients $(8 \%)$ would have experienced a total of eight IRs. Additionally, stopping after the occurrence of a single pre-infusion sUA level above $6 \mathrm{mg} / \mathrm{dl}$ would have further decreased this to just 547 infusions administered and two patients (2\%) having three total IRs (see Table 1).

The responder rates reflected in this change in the stopping rules are similar to the previous responder rate of $42 \%$ and unchanged from the application of post-infusion sUA stopping rules. Stopping after two consecutive pre-infusion sUA levels above $6 \mathrm{mg} / \mathrm{dl}$ would result in 35 patients remaining responders (41\%). Stopping pegloticase infusions after a single pre-infusion sUA level above $6 \mathrm{mg} / \mathrm{dl}$ would have resulted in $36 \%$ of patients remaining responders (31 out of 85) (see Table 1). 
Table 1 IRs and responders with post- and pre-infusion sUA discontinuation guidance

\begin{tabular}{|c|c|c|}
\hline Stopping rule & Patients with IRs out of $85, n(\%)$ & Responders to therapy out of $85, n(\%)$ \\
\hline None & $22(26)$ & $36(42)$ \\
\hline \multicolumn{3}{|c|}{ Post-infusion (Baraf et al. [3]) } \\
\hline $2 \mathrm{sUAs}>6 \mathrm{mg} / \mathrm{dl}$ & $12(14)$ & $35(41)$ \\
\hline $1 \mathrm{sUA}>6 \mathrm{mg} / \mathrm{dl}$ & $7(8)$ & $31(36)$ \\
\hline \multicolumn{3}{|c|}{ Pre-infusion (current analysis) } \\
\hline $2 \mathrm{sUAs}>6 \mathrm{mg} / \mathrm{dl}$ & $7(8)$ & $35(41)$ \\
\hline $1 \mathrm{sUA}>6 \mathrm{mg} / \mathrm{dl}$ & $2(2)$ & $31(36)$ \\
\hline
\end{tabular}

\section{DISCUSSION}

Upon approval and availability of pegloticase in 2011, there were concerns regarding its safety primarily related to the occurrence of IRs in the phase 3 trials. As outlined above, during the conduct of these trials it was not appreciated that the uric acid levels obtained at the time of pegloticase infusions could serve as a biomarker for the identification of patients with anti-pegloticase antibodies and that such antibodies were the cause of the increased risk for subsequent IRs.

Baraf et al. demonstrated the ability of sUA levels to identify patients at risk for IRs to pegloticase during therapy. In his analysis, uric acid levels were drawn with infusions and viewed 2 weeks later prior to the next infusion. In this current analysis, uric acid levels from the pivotal trials were reanalyzed to determine the IR rate if sUA levels had been available immediately prior to each pegloticase infusion instead of 2 weeks after. In contrast to the 22 patients experiencing IRs with no stopping rules or the 12 patients who would have experienced IRs if two post-infusion uric acid levels were utilized, we found that stopping pegloticase after two consecutive pre-infusion sUA levels above $6 \mathrm{mg} / \mathrm{dl}$ would have resulted in just seven patients having IRs. Similarly, stopping pegloticase therapy after the occurrence of a single pre-infusion sUA level above $6 \mathrm{mg} / \mathrm{dl}$ would have resulted in only two patients $(2 \%)$ experiencing three total IRs. The proportion of responders to pegloticase when applying preinfusion sUA stopping rules is unchanged from the prior analysis; stopping after two pre-infusion sUA levels above 6 results in 35 (41\%) patients remaining responders, while stopping after one pre-infusion sUA level above 6 results in $31(36 \%)$ patients remaining responders.

A limitation to this evaluation is that all sUA values in the study were drawn during pegloticase infusions, yet in this analysis it was extrapolated that they were drawn 1-2 days before each infusion. Additionally, it is important to note that these stopping rules do not apply to a patient's first pegloticase infusion.

In a new ongoing clinical trial, patients have shown that under this type of protocol it is logistically feasible to obtain uric acid levels 24-48 $\mathrm{h}$ prior to a scheduled pegloticase infusion and to withdraw a patient from treatment if a single pre-infusion sUA rises to greater than $6 \mathrm{mg} / \mathrm{dl}$ [5]. With this guidance for discontinuation of therapy in place, the rate of IRs has been $0.3 \%$ ( 1 out of 315 infusions), with one subject out of 50 ( $2 \%$ of patients) having a single mild IR [5]. This was the first prospective implementation of guidance for discontinuation of pegloticase in a clinical trial based on sUA levels while on therapy. The present analysis corroborated these findings looking retrospectively at the prior phase 3 trials of pegloticase. For patients on pegloticase therapy, the ability of pre-infusion sUA levels to identify patients who have lost efficacy from infusions and are therefore at risk of IRs appears to be a 
unique feature among biologic medications. For a commonly used, widely available, inexpensive blood test to reliably classify patients as ongoing therapeutic responders or non-responders prior to each infusion-allowing for therapy to be discontinued if warranted-is a powerful tool.

\section{CONCLUSIONS}

In patients on pegloticase therapy, uric acid level functions as a biomarker to identify patients who have developed anti-drug antibodies to pegloticase and are therefore at risk of IRs. By sampling and viewing pre-infusion sUA levels to determine whether to continue pegloticase therapy, the number of IRs that occur can be significantly reduced and the benefit of ongoing therapy validated.

\section{ACKNOWLEDGEMENTS}

The authors are appreciative of the participants who were involved in these trials.

Funding. Horizon Pharma funded this analysis and the journal's processing charges. All authors had full access to all of the data in this study and take complete responsibility for the integrity of the data and accuracy of the data analysis.

Authorship. All named authors meet the International Committee of Medical Journal Editors (ICMJE) criteria for authorship for this article, take responsibility for the integrity of the work as a whole, and have given their approval for this version to be published.

Disclosures. Brian LaMoreaux is an employee of and has stock in Horizon Pharma USA, Inc. Robert T. Keenan is a consultant and participated on advisory boards for Horizon. Herbert S. B. Baraf is a consultant for Horizon.

Compliance with Ethics Guidelines. This article is based on previously conducted studies and does not contain any studies with human participants or animals performed by any of the authors.

Data Availability. The datasets generated and analyzed during the current study are not publicly available but are available from the corresponding author on reasonable request.

Open Access. This article is distributed under the terms of the Creative Commons Attribution-NonCommercial 4.0 International License (http://creativecommons.org/licenses/ by-nc/4.0/), which permits any noncommercial use, distribution, and reproduction in any medium, provided you give appropriate credit to the original author(s) and the source, provide a link to the Creative Commons license, and indicate if changes were made.

\section{REFERENCES}

1. Sundy JS, Baraf HS, Yood RA, et al. Efficacy and tolerability of pegloticase for the treatment of chronic gout in patients refractory to conventional treatment: two randomized controlled trials. JAMA. 2011;306: 711-20.

2. Lipsky PE, Calabrese LH, Kavanaugh A, et al. Pegloticase immunogenicity: the relationship between efficacy and antibody development in patients treated for refractory chronic gout. Arthritis Res Ther. 2014;16:R60.

3. Baraf HS, Yood RA, Ottery FD, et al. Infusion-related reactions with pegloticase, a recombinant uricase for the treatment of chronic gout refractory to conventional therapy. J Clin Rheumatol. 2014;20:427-32.

4. Becker MA, Baraf HS, Yood RA, et al. Long-term safety of pegloticase in chronic gout refractory to conventional treatment. Ann Rheum Dis. 2013;72:1469-74.

5. Saag K, Feinman M, Kivitz AJ, Baraf HSB, Fleischmann R, Kavanaugh A, Lipsky PE. Initial Results of a Clinical Study to Determine Whether a Tolerizing Regimen of Pegloticase Can Increase the Frequency of Subjects Having Sustained Lowering of Serum Urate [abstract]. Arthritis Rheumatol. 2017;69(suppl 10). https:// acrabstracts.org/abstract/initial-results-of-a-clinicalstudy-to-determine-whether-a-tolerizing-regimenofpegloticase-can-increase-the-frequency-of-subjectshaving-sustained-lowering-of-serum-urate/. Accessed 12 Mar 2019. 